\title{
Information and education provision in bronchiectasis: co-development and evaluation of a novel patient-driven resource in a digital era
}

\author{
To the Editor:
}

Bronchiectasis presents a significant burden of disease for patients, their families and healthcare services, with recurrent hospital admissions and poorer health-related quality of life, in addition to higher rates of fatigue, depression and cardiovascular disease [1-3]. The increasing prevalence and burden of bronchiectasis is recognised worldwide [3-6]. Patient education is a fundamental part of long-term management planning, as highlighted in the new European Respiratory Society guidelines, yet there is a lack of information available to patients in comparison to other chronic respiratory conditions.

Lack of information has been identified as a potential barrier to treatment adherence and self-management in bronchiectasis $[7,8]$. In other chronic conditions, it has also been described that information must be presented and used in specific ways in order to produce change $[9,10]$. Involving patients and their families in the development process and understanding their information seeking and use can help to ensure that requirements are met $[11,12]$. The co-development of a high-quality, credible and engaging patient-driven resource for bronchiectasis could empower patients to recognise symptoms of exacerbations early, and equip them with the understanding, knowledge and skills required to self-manage effectively. Such improvements in patient understanding and self-management could facilitate improvements in disease stability and consequent reductions in healthcare service use.

We conducted a qualitative investigation of patients' needs and developed a patient-driven resource based upon these findings. Ethical approval was obtained from: NRES Committee South Central - Berkshire B (REC reference number 12/SC/0585) and UKCRN portfolio (UKCRN ID 115842).

Adult participants with bronchiectasis were recruited from across two sites in the north-east of England, incorporating one specialist bronchiectasis clinic and multiple general respiratory clinics. Purposeful sampling was used to achieve a maximum variation sample. Participants took part in a single, semi-structured interview to explore their unmet information needs and experiences of information use. Interviews were with either the patient alone $(n=8)$, or joint with a partner or family member $(n=9)$. Interview data was analysed using thematic analysis $[13,14]$, and identified four interlinking themes (to be reported in full elsewhere).

1) "Living your life with bronchiectasis": the experiences of patients and families living with bronchiectasis. Key points identified included the physical, psychological and social impacts of the condition, the nature of the disruption that a diagnosis of bronchiectasis presents and the uncertainties surrounding the future and prognosis.

2) "Developing support and coping mechanisms": ways in which patients and their families adapt to the disruptions that bronchiectasis imposes. Descriptions include tools used to enable them to cope with having the condition; and their support mechanisms, including medical teams, families and other patients with shared experiences.

3) "Taking back control and forming active partnerships with the medical team": further along the patient journey, patients began to take back some control and became able to recognise changes in their condition

@ERSpublications

Improvements in information for those living with bronchiectasis should use patient-driven codevelopment processes http://ow.ly/vWCB30iCn2M

Cite this article as: Hester KLM, Newton J, Rapley T, et al. Information and education provision in bronchiectasis: co-development and evaluation of a novel patient-driven resource in a digital era. Eur Respir J 2018; 51: 1702402 [https://doi.org/10.1183/13993003.02402-2017]. 
through experience. They still needed reassurance and advice from their medical team, but became active partners in their disease management.

4) "Connecting with information": participants' approaches and barriers to information seeking, use and interaction. This incorporated how, when and why users seek information, what formats and content were priorities and what would encourage engagement. Participants particularly highlighted the need for trustworthy, novel resources that were presented in keeping with their needs, including patients' perspectives.

A series of three co-development workshops were held with patients $(n=11)$ and family members $(n=4)$ (including both interviewed and additional participants) to facilitate translation of interview findings into a novel patient information resource. Workshops explored participant agreement or disagreement with the emergent themes and ideas, and concepts for resource design and content. A prototype was developed, then reviewed and altered. Experts in the management of bronchiectasis, independent reviewers and a media design company were involved throughout the co-development process to ensure the development of a high quality, accurate, professional and trustworthy resource.

A website containing both text and video content (www.bronchiectasis.me) and a 15-page A5 sized booklet were agreed to be the optimum formats. Features enabling users to ascertain credibility of the resources were identified and included having visible links with recognised health service providers and links with an academic institution. Preferred ways to group sections of information to facilitate ease of navigation were determined. The final version of the homepage was deemed to portray the required positive message that, generally, bronchiectasis is a condition that people can continue to live their lives with. Content was designed in keeping with interview findings and videos were filmed with professionals, patients and family members.

The resource was evaluated in a feasibility study (the BRIEF study, ISRCTN registry number: ISRCTN84229105, publication of results pending), identifying that participants found the resource useful (93\% of users). In addition, using a structured questionnaire, we detected a potential improvement in perceived understanding of bronchiectasis, its treatments and where to find more information amongst the intervention group. The BRIEF study also provided qualitative data which enabled confirmation of the effective use of the interview findings and co-development process, and facilitated further refinements to the resources. The use of web analytics identified that webpages viewed most frequently were in keeping with those identified within the interviews and co-development process to be most important to patients (diet and lifestyle advice, symptoms, diagnosis, and families and carers). Participants highlighted the usefulness of the video clips within the website, particularly those depicting other patients' stories.

A limitation of this resource development process was the single centre study design. The use of purposeful sampling within the recruitment process for the underpinning qualitative phase of this work went some way to mitigate this. There has been high worldwide demand for access to the site, with approximately 80000 page views since June 2014, confirming patients are seeking to access trustworthy bronchiectasis information resources.

Understanding how patients engage with information, in the context of their experiences of living with bronchiectasis, is fundamental when managing bronchiectasis with patients as partners. The use of qualitative methods within this study has facilitated a richer understanding of such experiences, including the nature of the biographical disruption that a diagnosis of bronchiectasis presents and the ways in which people manage this. In addition to gaining an understanding of patients' needs and developing information to meet those needs, prior literature supports the use of development processes that involve and are centred on potential users [12,15]. By having a user-driven development process, built upon a careful and analytical assessment of experiences, preferences and needs, a novel resource that was credible, met patients' requirements and encouraged engagement was produced.

This is the first report of a bronchiectasis information resource based upon in-depth qualitative exploration and understanding of the needs and experiences of patients and their families. The website (www.bronchiectasis.me) is now an open access resource undergoing further interactive developments, and the accompanying booklet "Living your life with bronchiectasis" has been used during development of the latest British Lung Foundation bronchiectasis patient information. This is the first step in using such a resource to allow equity of information access and dissemination of good practice, in order to positively influence patients' understanding, ability to self-manage and use of healthcare services. The approach used herein could be applied to other disease areas. To optimise engagement with, and the impact of, novel bronchiectasis information resources, patient-driven co-development processes should be used. The use of multiple formats, including video clips is important in an era of more digitally engaged patient populations. 
Katy L.M. Hester $\oplus^{1,2}$, Julia Newton ${ }^{3}$, Tim Rapley ${ }^{4}$, Vicky Ryan ${ }^{5}$ and Anthony De Soyza $\oplus^{1,2}$

${ }^{1}$ Institute of Cellular Medicine, Newcastle University, Newcastle upon Tyne, UK. ${ }^{2}$ Adult Bronchiectasis Service, Freeman Hospital, Newcastle upon Tyne Hospitals NHS Foundation Trust, Newcastle upon Tyne, UK. ${ }^{3}$ Royal Victoria Infirmary, Newcastle upon Tyne Hospitals NHS Foundation Trust, Newcastle upon Tyne, UK. ${ }^{4}$ Department of Social Work, Education and Community Wellbeing, Northumbria University, Newcastle upon Tyne, UK. ${ }^{5}$ Institute of Health and Society, Newcastle University, Newcastle upon Tyne, UK.

Correspondence: Katy L. M. Hester, Institute of Cellular Medicine, Newcastle University, Newcastle upon Tyne, NE2 4HH, UK. E-mail: katy.hester@ncl.ac.uk

Received: Nov 202017 | Accepted after revision: Feb 072018

Support statement: K.L.M. Hester is funded by a National Institute for Health Research Doctoral Research Fellowship (DRF-2012-05-149). This article presents independent work funded by the National Institute for Health Research (NIHR). The views expressed are those of the author(s) and not necessarily those of the NHS, the NIHR or the Department of Health. A. De Soyza acknowledges funding to the BronchUK network from the UK Medical Research Council (grant MR/L011263/1) and a prior Higher Education Funding Council (HEFCE senior lectureship). Funding information for this article has been deposited with the Crossref Funder Registry.

Conflict of interest: K.L.M. Hester reports grants from NIHR, during the conduct of the study. J. Newton reports grants from NIHR, during the conduct of the study. T. Rapley reports grants from NIHR, during the conduct of the study. A. De Soyza reports non-financial support from AstraZeneca (Bronchiectasis Interest Group meeting support), Novartis (in kind support for analysis in bronchiectasis) and Forest labs (Bronchiectasis Interest Group meeting support); personal fees from Bayer (speakers/advisory boards on bronchiectasis) and AstraZeneca (speakers/advisory boards on COPD); personal fees and other (advisory boards on bronchiectasis and in kind bench science support) from Novartis; other from Chiesi (travel bursary to attend ERS meeting), Almirall (travel bursary to attend ERS meeting) and Boehringer Ingelheim (travel bursary to attend BTS meeting); and grants from AstraZeneca (travel bursary to attend ATS meeting), all outside the submitted work. A. De Soyza has received medical education grant support for a UK bronchiectasis network from GSK, Gilead, Chiesi and Forest labs. A. De Soyza's employing institution receives fees for his work as coordinating investigator in a phase III trial in Bronchiectasis sponsored by Bayer.

Acknowledgements: We would like to acknowledge Holly Ainsworth for assistance with using statistical software for the analysis of the BRIEF study data, and the staff of the William Leech Centre for Lung Research at the Freeman Hospital in the recruitment and study visit conduct in the BRIEF study. We also thank the participants.

Author contributions: $\mathrm{KH}$ is the first and corresponding author of this article. TR, JN, VR and ADS were contributors to the manuscript and all authors read and approved the final manuscript.

\section{References}

1 Hester KLM, Macfarlane JG, Tedd H, et al. Fatigue in bronchiectasis. QJM 2012; 105: 235-240.

2 O'Leary CJ, Wilson CB, Hansell DM, et al. Relationship between psychological well-being and lung health status in patients with bronchiectasis. Respir Med 2002; 96: 686-692.

3 Navaratman V, Millett ER, Hurst JR, et al. Bronchiectasis and the risk of cardiovascular disease: a population-based study. Thorax 2017; 72: 161-166.

4 Quint JK, Millett ER, Joshi M, et al. Changes in the incidence, prevalence and mortality of bronchiectasis in the UK from 2004-2013: a population based cohort study. Eur Respir J 2016; 47: 186-193.

5 Ringshausen FC, de Roux A, Diel R, et al. Bronchiectasis in Germany: a population-based estimation of disease prevalence. Eur Respir J 2015; 46: 1805-1807.

6 Seitz AE, Olivier KN, Adjemian J, et al. Trends in bronchiectasis among medicare beneficiaries in the United States, 2000 to 2007. Chest 2012; 142: 432-439.

7 Lavery K, O’Neill B, Elborn JS, et al. Self-management in bronchiectasis: the patients' perspective. Eur Respir J 2007; 29: 541-547.

8 McCullough AR, Ryan C, O’Neill B, et al. Defining the content and delivery of an intervention to Change AdhereNce to treatment in BonchiEctasis (CAN-BE): A qualitative approach incorporating the Theoretical Domains Framework, behavioural change techniques and stakeholder expert panels. BMC Health Serv Res 2015; 15: 342 .

9 Becker MH. Theoretical models of adherence and strategies for improving adherence. In: S.A. Shumaker, ed. The Handbook of Health Behaviour Change. New York, Springer Publishing Company, 1990; pp. 5-43.

10 Gibson PG, Powell H, Coughlan J, et al. Self-management education and regular practitioner review for adults with asthma. Cochrane Database Syst Rev 2003; 1: CD001117.

11 Lambert SD, Loiselle CG. Health information seeking behaviour. Qual Health Res 2007; 17: 1006-1019.

12 Harland J, Bath P. Understanding the information behaviours of carers of people with dementia: A critical review of modelas from information science. Aging Ment Health 2008; 12: 467-477.

13 Gibbs G. Analyzing Qualitative Data. London, Sage, 2007.

14 Braun V, Clarke V. Using thematic analysis in psychology. Qual Res Psychol 2006; 3: 77-101.

15 Patient Information Forum. Making the case for information. 2013. www.pifonline.org.uk/wp-content/uploads/ 2013/05/PiF-full-report-FINAL-new.pdf 